\title{
Introduction to the Vol. 49, No. 1, 2022
}

\section{Maomi Ueno ${ }^{1}$}

Published online: 5 February 2022

(c) The Behaviormetric Society 2022

Welcome to the Vol. 49, No. 1, 2022 of Behaviormetrika. In this issue, we have the following five original papers and two notes. The original paper "Causal order identification to address confounding: binary variables" by Suzuki and Inaoka (2022) addresses an extension of the linear non-Gaussian acyclic model (LiNGAM) that determines the causal order among variables from a dataset when the variables are expressed by a set of linear equations, including noise. Specifically, they assume that the variables are binary. The existing LiNGAM assumes that no confounding is present, which is restrictive in practice. Based on the concept of independent component analysis (ICA), this paper proposes an extended framework in which the mutual information among the noises is minimized. Another significant contribution is to reduce the realization to the shortest path problem, in which the distance between each pair of nodes expresses an associated mutual information value, and the path with the minimum sum (KL divergence) is sought. Although $p$ ! mutual information values should be compared, this paper dramatically reduces the computation when no confounding is present. The proposed algorithm finds the globally optimal solution, while the existing approaches locally greedily seek the order based on hypothesis testing. We use the best estimator in the sense of Bayes/MDL that correctly detects independence for mutual information estimation. Experiments using artificial and actual data show that the proposed version of LiNGAM achieves significantly better performance, particularly when confounding is present.

The original paper "A confirmatory composite analysis for the Italian validation of the interactions anxiousness scale: a higher-order version"' by Ciavolino et al. (2021) examined the factor structure and model specifications of the Interaction Anxiousness Scale (IAS) with confirmatory composite analysis (CCA) PLS approach, that is to evaluate the presence of eventual sub-scales specific of Italian adolescents. Furthermore, they tested the measurement model of IAS with Italian adolescents using a higher order modeling, that is modeling the concept of social anxiousness on a more abstract dimension (referred as higher-order component) and its more concrete sub-dimensions (i.e. lower order components) linked by reflective relationships. The CCA and PLS-SEM results identified the reflective nature of

Maomi Ueno

ueno@ai.is.uec.ac.jp

1 University of Electro-Communications, Tokyo, Japan 
the IAS sub-scale scores, supporting an alternative measurement model of the IAS scores as a second-order reflective-reflective model.

The original paper "Observed score reliability indices in diagnostic classification models" by Yamaguchi and Templin (2021) derived observed score reliability indices based on diagnostic classification models as an extension of classical test theory-based reliability. Additionally, they derived conditional observed sum- and sub-score distributions. In this manner, various conditional expectations and conditional standard error of measurement estimates can be calculated for both sumand sub-scores of a test. The proposed methods provided a variety of expectations and standard errors for attribute estimates, which they demonstrated with an analysis of an empirical test. Moreover, a simulation study revealed the proposed sub-scorebased reliability index was correlated to a previously developed attribute mastery reliability index.

The original paper "Empirical analysis of a time-use model incorporating correlations among three household members" by Hasegawa and Ueda (2021) analyzed the living hours of three household members, who are fathers, mothers, and children, using micro-anonymous time-use data from the Japanese "Survey on Time Use and Leisure Activities." They used the data in the survey as compositional data, including zeros, and they estimated the living hours of the couples and their children using a Bayesian model incorporating not only the correlation among times spent on the various activities of each member but also the correlation among times spent on those of the three household members. The correlations were found between the activity time of household members such as commuting and housework time, showing that using a model incorporating the above correlations is important. Additionally, they calculated the partial effects, and the probability that the partial effects are positive using posterior results, and showed the effects of the household members' attributes on their time spent on activities.

The original paper "The effect of various simultaneous sources of mechanical error in the estimators of correlation causing deflation in reliability: seeking the best options of correlation for deflation-corrected reliability" by Jari Metsämuuronen (2022) addresses estimates of reliability by traditional estimators. They are deflated because the item-total or item-score correlation (Rit) or principal component- or factor loading $(\lambda i)$ embedded in the estimators are seriously deflated. Different optional estimators of correlation that can replace Rit and $\lambda i$ are compared in this article. Simulations show that estimators such as polychoric correlation (R PC), gamma $(G)$, dimension-corrected $G(G$ 2), and attenuation-corrected Rit (R AC) and eta (E AC) reflect the true correlation without any loss of information with several sources of technical or mechanical error in the estimators of correlation (MEC) including extreme item difficulty and item variance, small number of categories in the item and in the score, and the varying distributions of the latent variable. To obtain deflation corrected reliability, R PC, G, G 2, R AC, and E AC are likely to be the best options closely followed by r-bireg or r-polyreg coefficient (R REG).

The note "Robustness of factor solutions in exploratory factor analysis" by Goretzko and Bühner (2021) focus mainly on significance testing and confirmatory analyses, whereas exploratory analyses such as exploratory factor analysis are more or less ignored, although hardly any analysis has a comparable impact 
on entire research areas. Determining the correct number of factors for this analysis is probably the most crucial, yet ambiguous decision-especially since factor structures have often been not replicable. Hence, an approach based on bootstrapping the factor retention process is proposed to evaluate the robustness of factor retention criteria against sampling error and to predict whether a particular factor solution may be replicable. They used three samples of the "Big Five Structure Inventory" and four samples of the "10 Item Big Five Inventory" to illustrate the relationship between stable factor solutions across bootstrap samples and their replicability. In addition, they compared four factor retention criteria and an information criterion in terms of their stability on the one hand and their replicability on the other.

The note "Derivation of the percentile-based Tukey distributions" by Ptukhin et al. (2022) address the method of moments (MOM). The disadvantage of the MOM is that the estimates can be seriously biased, have large variance, or can be affected by outliers. The method of percentiles (MOP) provides a useful alternative to the MOM when the distributions are non-normal, specifically being more computationally efficient in terms of estimating population parameters. Examples include the third order power method and fifth order power method. Further, the $\mathrm{HH}, \mathrm{HR}$ and HQ distributions, as extensions of the Tukey $g-h(\mathrm{GH})$ family, are of interest for investigation using the MOP in this study. More specifically, closed form solutions are obtained for a skew function and a kurtosis function. A Monte Carlo simulation study which includes the comparison of monotonic and nonmonotonic transformation scenarios is also performed. The effect on Type I error and power rates under nonmonotonic scenarios are of special interest in the study. The empirical confirmation that rank transform is appropriate for $2 \times 2$ designs is also obtained.

Funding This research has no by any organization or individual.

\section{Declarations}

Conflict of interest The author declares no conflict of interest.

\section{References}

Ciavolino E, Ferrante L, Sternativo GA et al (2021) A confirmatory composite analysis for the Italian validation of the interactions anxiousness scale: a higher-order version. Behaviormetrika. https:// doi.org/10.1007/s41237-021-00151-X

Goretzko D, Bühner M (2021) Robustness of factor solutions in exploratory factor analysis. Behaviormetrika. https://doi.org/10.1007/s41237-021-00152-w

Hasegawa H, Ueda K (2022) Empirical analysis of a time-use model incorporating correlations among three household members. Behaviormetrika. https://doi.org/10.1007/s41237-021-00157-5

Metsämuuronen J (2022) The effect of various simultaneous sources of mechanical error in the estimators of correlation causing deflation in reliability: Seeking the best options of correlation for deflation-corrected reliability. Behaviormetrika (2022)

Ptukhin Y, Sheng Y, Headrick T (2022) Derivation of the percentile-based Tukey distributions. Behaviormetrika. https://doi.org/10.1007/s41237-021-00155-7 
Suzuki J, Inaoka Y (2021) Causal order identification to address confounding: binary variables. Behaviormetrika. https://doi.org/10.1007/s41237-021-00149-5

Yamaguchi K, Templin J (2021x) Observed score reliability indices in diagnostic classification models. Behaviormetrika. https://doi.org/10.1007/s41237-021-00153-9

Publisher's Note Springer Nature remains neutral with regard to jurisdictional claims in published maps and institutional affiliations. 